\title{
Arithmetic quantum chaos of Maass waveforms
}

\author{
H. Then
}

Abteilung Theoretische Physik, Universität Ulm, Albert-Einstein-Allee 11, 89069

Ulm, holger.then@physik.uni-ulm.de

Summary. We compute numerically eigenvalues and eigenfunctions of the quantum Hamiltonian that describes the quantum mechanics of a point particle moving freely in a particular three-dimensional hyperbolic space of finite volume and investigate the distribution of the eigenvalues.

\section{Introduction}

The distribution of the eigenvalues of a quantum Hamiltonian is a central subject that is studied in quantum chaos. There are some generally accepted conjectures about the nearest-neighbor spacing distributions of the eigenvalues.

Unless otherwise stated we use the following assumptions: The quantum mechanical system is desymmetrized with respect to all its unitary symmetries, and whenever we examine the distribution of the eigenvalues we regard them on the scale of the mean level spacings. Moreover, it is generically believed that after desymmetrization a generic quantum Hamiltonian possesses no degenerate eigenvalues.

Conjecture 1 (Berry, Tabor [1]). If the corresponding classical system is integrable, the eigenvalues behave like independent random variables and the distribution of the nearest-neighbor spacings is close to the Poisson distribution, i.e. there is no level repulsion.

Conjecture 2 (Bohigas, Giannoni, Schmit [2, 3]). If the corresponding classical system is chaotic, the eigenvalues are distributed like the eigenvalues of hermitian random matrices [4]. The corresponding ensembles depend only on the symmetries of the system:

- For chaotic systems without time-reversal invariance the distribution of the eigenvalues should be close to the distribution of the Gaussian Unitary Ensemble (GUE) which is characterized by a quadratic level repulsion. 
- For chaotic systems with time-reversal invariance and integer spin the distribution of the eigenvalues should be close to the distribution of the Gaussian Orthogonal Ensemble (GOE) which is characterized by a linear level repulsion.

- For chaotic systems with time-reversal invariance and half-integer spin the distribution of the eigenvalues should be close to the distribution of the Gaussian Symplectic Ensemble (GSE) which is characterized by a quartic level repulsion.

These conjectures are very well confirmed by numerical calculations, but several exceptions are known. Here are two examples:

Exception 1 The harmonic oscillator is classically integrable, but its spectrum is equidistant.

Exception 2 The geodesic motion on surfaces with constant negative curvature provides a prime example for classical chaos. In some cases, however, the nearest-neighbor distribution of the eigenvalues of the Laplacian on these surfaces appears to be Poissonian.

"A strange arithmetical structure of chaos" in the case of surfaces of constant negative curvature that are generated by arithmetic fundamental groups was discovered by Aurich and Steiner [5], see also Aurich, Bogomolny, and Steiner [6. Deviations from the expected GOE-behaviour in the case of a particular arithmetic surface were numerically observed by Bohigas, Giannoni, and Schmit [3] and by Aurich and Steiner [7. Computations coming out in 7,8 showed, however, that the level statistics on 30 generic (i.e. non-arithmetic) surfaces were in nice agreement with the expected random-matrix theory prediction in accordance with conjecture 2 This has led Bogomolny, Georgeot, Giannoni, and Schmit 9], Bolte, Steil, and Steiner [10, and Sarnak 11] to introduce the concept of arithmetic quantum chaos.

Conjecture 3 (Arithmetic Quantum Chaos). On surfaces of constant negative curvature that are generated by arithmetic fundamental groups, the distribution of the eigenvalues of the quantum Hamiltonian are close to the Poisson distribution. Due to level clustering small spacings occur comparably often.

We compute numerically the eigenvalues and eigenfunctions of the Laplacian that describes the quantum mechanics of a point particle moving freely in the non-integrable three-dimensional hyperbolic space of constant negative curvature generated by the Picard group. The Picard group is arithmetic and we find that our results are in accordance with the conjecture of arithmetic quantum chaos.

For the definition of an arithmetic group we refer the reader to [12]. 


\section{Preliminaries: The modular group}

For simplicity we first introduce the topology and geometry of the twodimensional surface of constant negative curvature that is generated by the modular group [13. It will then be easy to carry over to the three-dimensional space of constant negative curvature that is generated by the Picard group.

The construction begins with the upper half-plane,

$$
\mathcal{H}=\left\{(x, y) \in \mathbb{R}^{2} ; \quad y>0\right\},
$$

equipped with the hyperbolic metric of constant negative curvature

$$
d s^{2}=\frac{d x^{2}+d y^{2}}{y^{2}} .
$$

A free particle on the upper half-plane moves along geodesics, which are straight lines and semicircles perpendicular to the $x$-axis, respectively, see figure 1

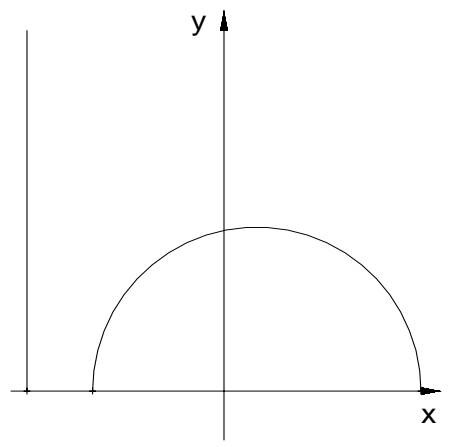

Fig. 1. Geodesics in the upper half-plane of constant negative curvature.

Expressing a point $(x, y) \in \mathcal{H}$ as a complex number $z=x+\mathrm{i} y$, all isometries of the hyperbolic metric are given by the group of linear fractional transformations,

$$
z \mapsto \gamma z=\frac{a z+b}{c z+d} ; \quad a, b, c, d \in \mathbb{R}, \quad a d-b c=1,
$$

which is isomorphic to the group of matrices

$$
\gamma=\left(\begin{array}{ll}
a & b \\
c & d
\end{array}\right) \in \mathrm{SL}(2, \mathbb{R})
$$

up to a common sign of the matrix entries, 


$$
\mathrm{SL}(2, \mathbb{R}) /\{ \pm 1\}=\operatorname{PSL}(2, \mathbb{R}) .
$$

In analogy to the concept of a fundamental cell in a regular lattice of a crystal we can introduce a fundamental domain of a discrete group $\Gamma \subset$ $\operatorname{PSL}(2, \mathbb{R})$.

Definition 1. A fundamental domain of the discrete group $\Gamma$ is an open subset $\mathcal{F} \subset \mathcal{H}$ with the following conditions: The closure of $\mathcal{F}$ meets each orbit $\Gamma z=\{\gamma z ; \gamma \in \Gamma\}$ at least once, $\mathcal{F}$ meets each orbit $\Gamma z$ at most once, and the boundary of $\mathcal{F}$ has Lebesgue measure zero.

If we choose the group $\Gamma$ to be the modular group,

$$
\Gamma=\operatorname{PSL}(2, \mathbb{Z}),
$$

which is generated by a translation and an inversion,

$$
\begin{gathered}
\left(\begin{array}{ll}
1 & 1 \\
0 & 1
\end{array}\right): z \mapsto z+1, \\
\left(\begin{array}{cc}
0 & -1 \\
1 & 0
\end{array}\right): z \mapsto-z^{-1},
\end{gathered}
$$

the fundamental domain of standard shape is

$$
\mathcal{F}=\left\{z=x+\mathrm{i} y \in \mathcal{H} ; \quad-\frac{1}{2}<x<\frac{1}{2}, \quad|z|>1\right\},
$$

see figure 2. The isometric copies of the fundamental domain $\gamma \mathcal{F}, \gamma \in \Gamma$,

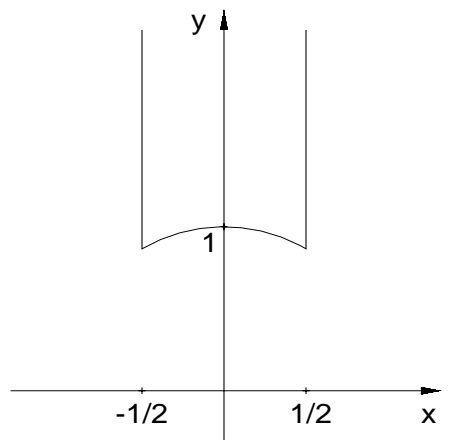

Fig. 2. The fundamental domain of the modular group.

tessellate the upper half-plane completely without any overlap or gap, see figure 3 


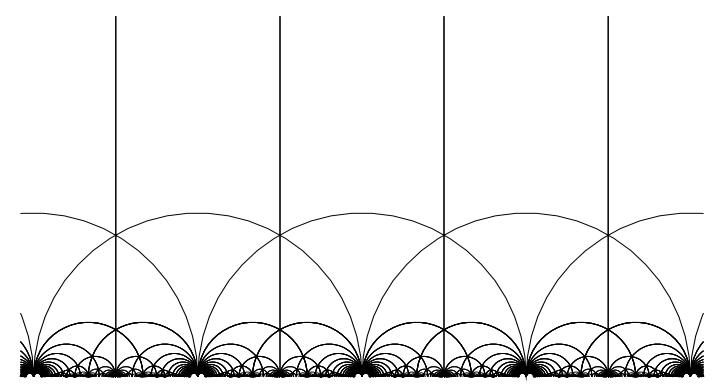

Fig. 3. The upper half-plane tessellated with isometric copies of the fundamental domain.

Identifying the fundamental domain $\mathcal{F}$ and parts of its boundary with all its isometric copies $\gamma \mathcal{F}, \forall \gamma \in \Gamma$, defines the topology to be the quotient space $\Gamma \backslash \mathcal{H}$. The quotient space $\Gamma \backslash \mathcal{H}$ can also be thought of as the fundamental domain $\mathcal{F}$ with its faces glued according to the elements of the group $\Gamma$, see figure 4

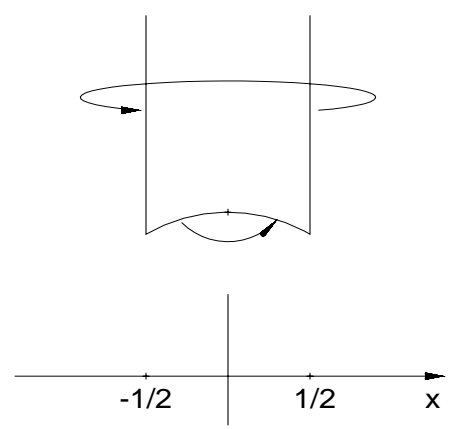

Fig. 4. Identifying the faces of the fundamental domain according to the elements of the modular group.

Any function being defined on the upper half-plane which is invariant under linear fractional transformations,

$$
f(z)=f(\gamma z) \quad \forall \gamma \in \Gamma
$$

can be identified with a function living on the quotient space $\Gamma \backslash \mathcal{H}$. A function on the quotient space is tantamount to a function on the fundamental domain 
with periodic boundary conditions. Vice versa, any function being defined on the quotient space can be identified with an automorphic function, $f(z)=$ $f(\gamma z), \forall \gamma \in \Gamma$, living on the upper half-plane.

With the hyperbolic metric the quotient space $\Gamma \backslash \mathcal{H}$ inherits the structure of an orbifold. An orbifold locally looks like a manifold, with the exception that it is allowed to have elliptic fix-points.

The orbifold of the modular group has one parabolic and two elliptic fixpoints,

$$
z=\mathrm{i} \infty, \quad z=\mathrm{i}, \quad \text { and } \quad z=\frac{1}{2}+\mathrm{i} \frac{\sqrt{3}}{2} .
$$

The parabolic one fixes a cusp at $z=\mathrm{i} \infty$ which is invariant under the parabolic element

$$
\left(\begin{array}{ll}
1 & 1 \\
0 & 1
\end{array}\right)
$$

Hence the orbifold of the modular group is non-compact. The volume element corresponding to the hyperbolic metric reads

$$
d \mu=\frac{d x d y}{y^{2}}
$$

such that the volume of the orbifold $\Gamma \backslash \mathcal{H}$ is finite,

$$
\operatorname{vol}(\Gamma \backslash \mathcal{H})=\frac{\pi}{3} .
$$

Scaling the units such that $\hbar=1$ and $2 m=1$, the stationary Schrödinger equation which describes the quantum mechanics of a point particle moving freely in the orbifold $\Gamma \backslash \mathcal{H}$ becomes

$$
(\Delta+\lambda) f(z)=0,
$$

where the hyperbolic Laplacian is given by

$$
\Delta=y^{2}\left(\frac{\partial^{2}}{\partial x^{2}}+\frac{\partial^{2}}{\partial y^{2}}\right)
$$

and $\lambda$ is the scaled energy. We can relate the the eigenvalue problem defined on the orbifold $\Gamma \backslash \mathcal{H}$ to the eigenvalue problem defined on the upper-half space, with the eigenfunctions being subject to the automorphy condition relative to the discrete group $\Gamma$,

$$
f(\gamma z)=f(z) \quad \forall \gamma \in \Gamma .
$$

In order to avoid solutions that grow exponentially in the cusp, we impose the boundary condition 


$$
f(z)=O\left(y^{\kappa}\right) \quad \text { for } \quad z \rightarrow \mathrm{i} \infty
$$

where $\kappa$ is some positive constant.

The solutions of this eigenvalue problem can be identified with Maass waveforms [14. The identification is worthwhile, since much is known about Maass waveforms from number theory and harmonic analysis which will simplify their computation, see e.g. [15, 16, 17, 18, 19, 20, 13, 21, 22, 23].

\section{The Picard group}

In the three-dimensional case one considers the upper-half space,

$$
\mathcal{H}=\left\{\left(x_{0}, x_{1}, y\right) \in \mathbb{R}^{3} ; \quad y>0\right\}
$$

equipped with the hyperbolic metric

$$
d s^{2}=\frac{d x_{0}^{2}+d x_{1}^{2}+d y^{2}}{y^{2}} .
$$

The geodesics of a particle moving freely in the upper half-space are straight lines and semicircles perpendicular to the $x_{0}-x_{1}$-plane, respectively, see figure 5.

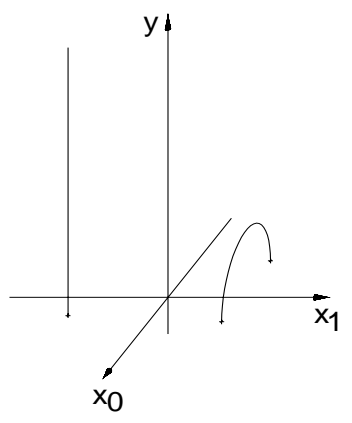

Fig. 5. Geodesics in the upper half-space of constant negative curvature.

Expressing any point $\left(x_{0}, x_{1}, y\right) \in \mathcal{H}$ as a Hamilton quaternion, $z=x_{0}+$ $\mathrm{i} x_{1}+\mathrm{j} y$, with the multiplication defined by $\mathrm{i}^{2}=-1, \mathrm{j}^{2}=-1, \mathrm{ij}+\mathrm{ji}=0$, all motions in the upper half-space are given by linear fractional transformations

$$
z \mapsto \gamma z=(a z+b)(c z+d)^{-1} ; \quad a, b, c, d \in \mathbb{C}, \quad a d-b c=1 .
$$

The group of these transformations is isomorphic to the group of matrices 


$$
\gamma=\left(\begin{array}{ll}
a & b \\
c & d
\end{array}\right) \in \mathrm{SL}(2, \mathbb{C})
$$

up to a common sign of the matrix entries,

$$
\operatorname{SL}(2, \mathbb{C}) /\{ \pm 1\}=\operatorname{PSL}(2, \mathbb{C}) .
$$

The motions provided by the elements of $\operatorname{PSL}(2, \mathbb{C})$ exhaust all orientation preserving isometries of the hyperbolic metric on $\mathcal{H}$.

Remark 1. If one wants to avoid using quaternions, the point $\left(x_{0}, x_{1}, y\right) \in \mathcal{H}$ can be expressed by $(x, y) \in \mathbb{C} \times \mathbb{R}$ with $x=x_{0}+\mathrm{i} x_{1}$ and $y>0$. But then the linear fractional transformation look somewhat more complicated,

$$
(x, y) \mapsto \gamma(x, y)=\left(\frac{(a x+b)(\bar{c} \bar{x}+\bar{d})+a \bar{c} y^{2}}{|c x+d|^{2}+|c y|^{2}}, \frac{y}{|c x+d|^{2}+|c y|^{2}}\right) .
$$

In order to keep the notation simple we hence use quaternions.

We now choose the discrete group $\Gamma \subset \operatorname{PSL}(2, \mathbb{C})$ generated by the cosets of three elements,

$$
\left(\begin{array}{ll}
1 & 1 \\
0 & 1
\end{array}\right), \quad\left(\begin{array}{ll}
1 & \mathrm{i} \\
0 & 1
\end{array}\right), \quad\left(\begin{array}{cc}
0 & -1 \\
1 & 0
\end{array}\right)
$$

which yield two translations and one inversion,

$$
z \mapsto z+1, \quad z \mapsto z+\mathrm{i}, \quad z \mapsto-z^{-1} .
$$

This group $\Gamma$ is called the Picard group. The three motions generating $\Gamma$, together with the coset of the element

$$
\left(\begin{array}{cc}
\mathrm{i} & 0 \\
0 & -\mathrm{i}
\end{array}\right)
$$

that is isomorphic to the symmetry

$$
z=x+\mathrm{j} y \mapsto \mathrm{i} z \mathrm{i}=-x+\mathrm{j} y,
$$

can be used to construct the fundamental domain of standard shape

$$
\mathcal{F}=\left\{z=x_{0}+\mathrm{i} x_{1}+\mathrm{j} y \in \mathcal{H} ; \quad-\frac{1}{2}<x_{0}<\frac{1}{2}, \quad 0<x_{1}<\frac{1}{2}, \quad|z|>1\right\},
$$

see figure [6] Identifying the faces of the fundamental domain according to the elements of the group $\Gamma$ leads to a realization of the quotient space $\Gamma \backslash \mathcal{H}$, see figure 7

With the hyperbolic metric the quotient space $\Gamma \backslash \mathcal{H}$ inherits the structure of an orbifold that has one parabolic and four elliptic fix-points, 


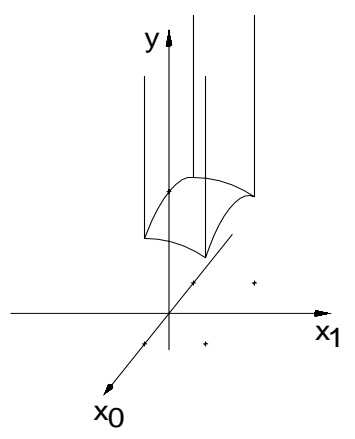

Fig. 6. The fundamental domain of the Picard group.

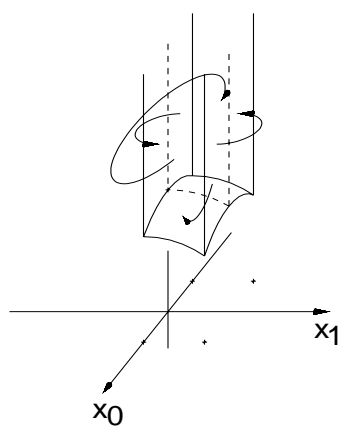

Fig. 7. Identifying the faces of the fundamental domain according to the elements of the Picard group.

$$
z=\mathrm{j} \infty, \quad z=\mathrm{j}, \quad z=\frac{1}{2}+\mathrm{j} \sqrt{\frac{3}{4}}, \quad z=\frac{1}{2}+\mathrm{i} \frac{1}{2}+\mathrm{j} \sqrt{\frac{1}{2}}, \quad z=\mathrm{i} \frac{1}{2}+\mathrm{j} \sqrt{\frac{3}{4}}
$$

The parabolic fix-point corresponds to a cusp at $z=\mathrm{j} \infty$ that is invariant under the parabolic elements

$$
\left(\begin{array}{ll}
1 & 1 \\
0 & 1
\end{array}\right), \quad \text { and } \quad\left(\begin{array}{ll}
1 & \mathrm{i} \\
0 & 1
\end{array}\right)
$$

The volume element deriving from the hyperbolic metric reads

$$
d \mu=\frac{d x_{0} d x_{1} d y}{y^{3}}
$$

such that the volume of the non-compact orbifold $\Gamma \backslash \mathcal{H}$ is finite [24],

$$
\operatorname{vol}(\Gamma \backslash \mathcal{H})=\frac{\zeta_{K}(2)}{4 \pi^{2}} \simeq 0.305
$$


where

$$
\zeta_{K}(s)=\frac{1}{4} \sum_{\nu \in \mathbb{Z}[\mathrm{i}]-\{0\}}(\nu \bar{\nu})^{-s}, \quad \Re s>1,
$$

is the Dedekind zeta function.

We are interested in the eigenfunctions of the Laplacian,

$$
\Delta=y^{2}\left(\frac{\partial^{2}}{\partial x_{0}^{2}}+\frac{\partial^{2}}{\partial x_{1}^{2}}+\frac{\partial^{2}}{\partial y^{2}}\right)-y \frac{\partial}{\partial y},
$$

which determine the quantum mechanics of a particle moving freely in the orbifold $\Gamma \backslash \mathcal{H}$. As in the preceding section we identify the solutions with Maass waveforms 25$]$.

Since the Maass waveforms are automorphic, and therefore periodic in $x_{0}$ and $x_{1}$, it follows that they can be expanded into a Fourier series,

$$
f(z)=u(y)+\sum_{\beta \in \mathbb{Z}[\mathrm{i}]-\{0\}} a_{\beta} y K_{\mathrm{i} r}(2 \pi|\beta| y) \mathrm{e}^{2 \pi \mathrm{i} \Re \beta x},
$$

where

$$
u(y)= \begin{cases}b_{0} y^{1+\mathrm{i} r}+b_{1} y^{1-\mathrm{i} r} & \text { if } r \neq 0, \\ b_{2} y+b_{3} y \ln y & \text { if } r=0 .\end{cases}
$$

$K_{\mathrm{i} r}(x)$ is the K-Bessel function whose order is connected with the eigenvalue $\lambda$ by

$$
\lambda=r^{2}+1
$$

If a Maass waveform vanishes in the cusp,

$$
\lim _{z \rightarrow \mathrm{j} \infty} f(z)=0
$$

it is called a Maass cusp form. Maass cusp forms are square integrable over the fundamental domain, $\langle f, f\rangle<\infty$, where

$$
\langle f, g\rangle=\int_{\Gamma \backslash \mathcal{H}} \bar{f} g d \mu
$$

is the Petersson scalar product.

According to the Roelcke-Selberg spectral resolution of the Laplacian 16. 17, its spectrum contains both a discrete and a continuous part. The discrete part is spanned by the constant eigenfunction $f_{0}$ and a countable number of Maass cusp forms $f_{1}, f_{2}, f_{3}, \ldots$ which we take to be ordered with increasing eigenvalues, $0=\lambda_{0}<\lambda_{1} \leq \lambda_{2} \leq \lambda_{3} \leq \ldots$. The continuous part of the spectrum $\lambda \geq 1$ is spanned by the Eisenstein series $E(x, 1+\mathrm{i} r)$ which are 
known analytically [26, 27]. The Fourier coefficients of the functions $\Lambda_{K}(1+$ $\mathrm{i} r) E(x, 1+\mathrm{i} r)$ are given by

$$
b_{0}=\Lambda_{K}(1+\mathrm{i} r), \quad b_{1}=\Lambda_{K}(1-\mathrm{i} r), \quad a_{\beta}=2 \sum_{\substack{\lambda, \mu \in \mathbb{Z}[\mathrm{i}] \\ \lambda \mu=\beta}}\left|\frac{\lambda}{\mu}\right|^{\mathrm{i} r},
$$

where

$$
\Lambda_{K}(s)=4 \pi^{-s} \Gamma(s) \zeta_{K}(s)
$$

has an analytic continuation into the complex plane except for a pole at $s=1$.

Normalizing the Maass cusp forms according to

$$
\left\langle f_{n}, f_{n}\right\rangle=1
$$

we can expand any square integrable function $\phi \in L^{2}(\Gamma \backslash \mathcal{H})$ in terms of Maass waveforms, 28,

$$
\phi(z)=\sum_{n \geq 0}\left\langle f_{n}, \phi\right\rangle f_{n}(z)+\frac{1}{2 \pi \mathrm{i}} \int_{\Re s=1}\langle E(\cdot, s), \phi\rangle E(z, s) d s .
$$

The eigenvalues and their associated Maass cusp forms are not known analytically. Thus, one has to approximate them numerically. Previous calculations of eigenvalues for the Picard group can be found in [29, 30, 31, 32. By making use of the Hecke operators [29, 33] and the multiplicative relations among the coefficients, Steil 32 obtained a non-linear system of equations which allowed him to compute 2545 consecutive eigenvalues. We extend these computations with the use of Hejhal's algorithm [34.

\section{Hejhal's algorithm}

Hejhal found a linear stable algorithm for computing Maass waveforms together with their eigenvalues which he used for groups acting on the twodimensional hyperbolic plane [34, see also 35, 36. We make use of this algorithm which is based on the Fourier expansion and the automorphy condition. We apply it for the Picard group acting on the three-dimensional hyperbolic space. For the Picard group no small eigenvalues $0<\lambda=r^{2}+1<1$ exist [37. Therefore, $r$ is real and the term $u(y)$ in the Fourier expansion of Maass cusp forms vanishes. Due to the exponential decay of the K-Bessel function for large arguments (12) and the polynomial bound of the coefficients 25,

$$
a_{\beta}=O(|\beta|), \quad|\beta| \rightarrow \infty,
$$

the absolutely convergent Fourier expansion can be truncated, 


$$
f(z)=\sum_{\substack{\beta \in \mathbb{Z}[\mathrm{i}]-\{0\} \\|\beta| \leq M}} a_{\beta} y K_{\mathrm{i} r}(2 \pi|\beta| y) \mathrm{e}^{2 \pi \mathrm{i} \Re \beta x}+[[\varepsilon]]
$$

if we bound $y$ from below. Given $\varepsilon>0, r$, and $y$, we determine the smallest $M=M(\varepsilon, r, y)$ such that the inequalities

$$
2 \pi M y \geq r \quad \text { and } \quad K_{\mathrm{i} r}(2 \pi M y) \leq \varepsilon \max _{x}\left(K_{\mathrm{i} r}(x)\right)
$$

hold. Larger $y$ allow smaller $M$. In all truncated terms,

$$
[[\varepsilon]]=\sum_{\substack{\beta \in \mathbb{Z}[\mathrm{i}]-\{0\} \\|\beta|>M}} a_{\beta} y K_{\mathrm{i} r}(2 \pi|\beta| y) \mathrm{e}^{2 \pi \mathrm{i} \Re \beta x},
$$

the K-Bessel function decays exponentially in $|\beta|$, and already the K-Bessel function of the first truncated summand is smaller than $\varepsilon$ times most of the $\mathrm{K}$ Bessel functions in the sum of (2). Thus, the error $[[\varepsilon]]$ does at most marginally exceed $\varepsilon$. The reason why $[[\varepsilon]]$ can exceed $\varepsilon$ somewhat is due to the possibility that the summands in (2) cancel each other, or that the coefficients in the truncated terms are larger than in (2). By a finite two-dimensional Fourier transformation the Fourier expansion (2) is solved for its coefficients

$$
a_{\gamma} y K_{\mathrm{i} r}(2 \pi|\gamma| y)=\frac{1}{(2 Q)^{2}} \sum_{x \in \mathbb{X}[\mathrm{i}]} f(x+\mathrm{j} y) \mathrm{e}^{-2 \pi \mathrm{i} \Re \gamma x}+[[\varepsilon]],
$$

where $\mathbb{X}[\mathrm{i}]$ is a two-dimensional equally distributed set of $(2 Q)^{2}$ numbers,

$$
\mathbb{X}[\mathrm{i}]=\left\{\frac{k_{0}+\mathrm{i} k_{1}}{2 Q} ; \quad k_{i}=-Q+\frac{1}{2},-Q+\frac{3}{2}, \ldots, Q-\frac{3}{2}, Q-\frac{1}{2}, \quad i=0,1\right\},
$$

with $2 Q>M+|\gamma|$.

By automorphy we have

$$
f(z)=f\left(z^{*}\right)
$$

where $z^{*}$ is the $\Gamma$-pullback of the point $z$ into the fundamental domain $\mathcal{F}$,

$$
z^{*}=\gamma z, \quad \gamma \in \Gamma, \quad z^{*} \in \mathcal{F}
$$

Thus, a Maass cusp form can be approximated by

$$
f(x+\mathrm{j} y)=f\left(x^{*}+\mathrm{j} y^{*}\right)=\sum_{\substack{\beta \in \mathbb{Z}[\mathrm{i}]-\{0\} \\|\beta| \leq M_{0}}} a_{\beta} y^{*} K_{\mathrm{i} r}\left(2 \pi|\beta| y^{*}\right) \mathrm{e}^{2 \pi \mathrm{i} \Re \beta x^{*}}+[[\varepsilon]],
$$

where $y^{*}$ is always larger or equal than the height $y_{0}$ of the lowest points of the fundamental domain $\mathcal{F}$, 


$$
y_{0}=\min _{z \in \mathcal{F}}(y)=\frac{1}{\sqrt{2}}
$$

allowing us to replace $M(\varepsilon, r, y)$ by $M_{0}=M\left(\varepsilon, r, y_{0}\right)$.

Choosing $y$ smaller than $y_{0}$ the $\Gamma$-pullback $z \mapsto z^{*}$ of any point into the fundamental domain $\mathcal{F}$ makes at least once use of the inversion $z \mapsto-z^{-1}$, possibly together with the translations $z \mapsto z+1$ and $z \mapsto z+\mathrm{i}$. This is called implicit automorphy, since it guarantees the invariance $f(z)=f\left(-z^{-1}\right)$. The conditions $f(z)=f(z+1)$ and $f(z)=f(z+\mathrm{i})$ are automatically satisfied due to the Fourier expansion.

Making use of the implicit automorphy by replacing $f(x+\mathrm{j} y)$ in (3) with the right-hand side of (4) gives

$a_{\gamma} y K_{\mathrm{i} r}(2 \pi|\gamma| y)=\frac{1}{(2 Q)^{2}} \sum_{x \in \mathbb{X}[\mathrm{i}]} \sum_{\substack{\beta \in \mathbb{Z}[\mathrm{i}]-\{0\} \\|\beta| \leq M_{0}}} a_{\beta} y^{*} K_{\mathrm{i} r}\left(2 \pi|\beta| y^{*}\right) \mathrm{e}^{2 \pi \mathrm{i} \Re \beta x^{*}} \mathrm{e}^{-2 \pi \mathrm{i} \Re \gamma x}+[[2 \varepsilon]]$,

which is the central identity in the algorithm.

The symmetry in the Picard group and the symmetries of the fundamental domain imply that the Maass waveforms fall into four symmetry classes 32 named $\mathbf{D}, \mathbf{G}, \mathbf{C}$, and $\mathbf{H}$, satisfying

$$
\begin{array}{ll}
\mathbf{D}: & f(x+\mathrm{j} y)=f(\mathrm{i} x+\mathrm{j} y)=f(-\bar{x}+\mathrm{j} y), \\
\mathbf{G}: & f(x+\mathrm{j} y)=f(\mathrm{i} x+\mathrm{j} y)=-f(-\bar{x}+\mathrm{j} y), \\
\mathbf{C}: & f(x+\mathrm{j} y)=-f(\mathrm{i} x+\mathrm{j} y)=f(-\bar{x}+\mathrm{j} y), \\
\mathbf{H}: & f(x+\mathrm{j} y)=-f(\mathrm{i} x+\mathrm{j} y)=-f(-\bar{x}+\mathrm{j} y),
\end{array}
$$

respectively, see figure 8 from which the symmetry relations among the coefficients follow,

$$
\begin{array}{ll}
\mathbf{D}: & a_{\beta}=a_{\mathrm{i} \beta}=a_{\bar{\beta}}, \\
\mathbf{G}: & a_{\beta}=a_{\mathrm{i} \beta}=-a_{\bar{\beta}}, \\
\mathbf{C}: & a_{\beta}=-a_{\mathrm{i} \beta}=a_{\bar{\beta}}, \\
\mathbf{H}: & a_{\beta}=-a_{\mathrm{i} \beta}=-a_{\bar{\beta}} .
\end{array}
$$

Defining

$$
\operatorname{cs}(\beta, x)=\sum_{\sigma \in \mathbb{S}_{\beta}} s_{\sigma \beta} \mathrm{e}^{2 \pi \mathrm{i} \Re \sigma x}
$$

where $s_{\sigma \beta}$ is given by

$$
a_{\sigma}=s_{\sigma \beta} a_{\beta}
$$

and 

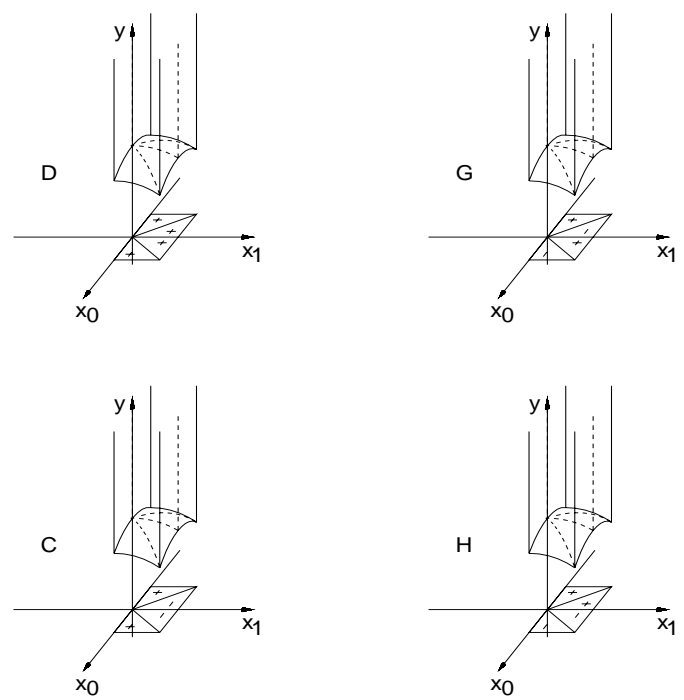

Fig. 8. The symmetries $\mathbf{D}, \mathbf{G}, \mathbf{C}$, and $\mathbf{H}$ from top left to bottom right.

$$
\sigma \in \mathbb{S}_{\beta}= \begin{cases}\{\beta, \mathrm{i} \beta,-\beta,-\mathrm{i} \beta, \bar{\beta}, \mathrm{i} \bar{\beta},-\bar{\beta},-\mathrm{i} \bar{\beta}\} & \text { if } \bar{\beta} \notin\{\beta, \mathrm{i} \beta,-\beta,-\mathrm{i} \beta\} \\ \{\beta, \mathrm{i} \beta,-\beta,-\mathrm{i} \beta\} & \text { else, }\end{cases}
$$

the Fourier expansion (1) of the Maass waveforms can be written

$$
f(z)=u(y)+\sum_{\beta \in \tilde{\mathbb{Z}}[\mathrm{i}]-\{0\}} a_{\beta} y K_{\mathrm{i} r}(2 \pi|\beta| y) \operatorname{cs}(\beta, x),
$$

where the tilde operator on a set of numbers is defined such that

$$
\tilde{\mathbb{X}} \subset \mathbb{X}, \quad \bigcup_{x \in \tilde{\mathbb{X}}} \mathbb{S}_{x}=\mathbb{X}, \quad \text { and } \bigcap_{x \in \tilde{\mathbb{X}}} \mathbb{S}_{x}=\emptyset
$$

holds.

Forgetting about the error $[[2 \varepsilon]]$ the set of equations (5) can be written as

$$
\sum_{\substack{\beta \in \tilde{\mathbb{Z}}[\mathrm{i}]-\{0\} \\|\beta| \leq M_{0}}} V_{\gamma \beta}(r, y) a_{\beta}=0, \quad \gamma \in \tilde{\mathbb{Z}}[\mathrm{i}]-\{0\}, \quad|\gamma| \leq M_{0},
$$

where the matrix $V=\left(V_{\gamma \beta}\right)$ is given by

$V_{\gamma \beta}(r, y)=\#\left\{\sigma \in \mathbb{S}_{\gamma}\right\} y K_{\mathrm{i} r}(2 \pi|\gamma| y) \delta_{\gamma \beta}-\frac{1}{(2 Q)^{2}} \sum_{x \in \mathbb{X}[\mathrm{i}]} y^{*} K_{\mathrm{i} r}\left(2 \pi|\beta| y^{*}\right) \operatorname{cs}\left(\beta, x^{*}\right) \operatorname{cs}(\gamma,-x)$. 
Since $y<y_{0}$ can always be chosen such that $K_{\mathrm{ir}}(2 \pi|\gamma| y)$ is not too small, the diagonal terms in the matrix $V$ do not vanish for large $|\gamma|$ and the matrix is well conditioned.

We are now looking for the non-trivial solutions of (6) for $1 \leq|\gamma| \leq M_{0}$ that simultaneously give the eigenvalues $\lambda=r^{2}+1$ and the coefficients $a_{\beta}$. Trivial solutions are avoided by setting one of the coefficients equal to one, $a_{\alpha}=1$. Here we choose $\alpha$ to be $1,2+\mathrm{i}, 1$, and $1+\mathrm{i}$, for the symmetry classes $\mathbf{D}, \mathbf{G}, \mathbf{C}$, and $\mathbf{H}$, respectively.

Since the eigenvalues are unknown we discretize the $r$ axis and solve for each $r$ value on this grid the inhomogeneous system of equations

$$
\sum_{\substack{\beta \in \tilde{\mathbb{Z}}[\mathrm{i}]-\{0, \alpha\} \\|\beta| \leq M_{0}}} V_{\gamma \beta}\left(r, y^{\# 1}\right) a_{\beta}=-V_{\gamma \alpha}\left(r, y^{\# 1}\right), \quad 1 \leq|\gamma| \leq M_{0},
$$

where $y^{\# 1}<y_{0}$ is chosen such that $K_{\text {ir }}\left(2 \pi|\gamma| y^{\# 1}\right)$ is not too small for $1 \leq$ $|\gamma| \leq M_{0}$. A good value to try for $y^{\# 1}$ is given by $2 \pi M_{0} y^{\# 1}=r$.

It is important to check whether

$$
g_{\gamma}=\sum_{\substack{\beta \in \tilde{\mathbb{Z}}[\mathrm{i}]-\{0\} \\|\beta| \leq M_{0}}} V_{\gamma \beta}\left(r, y^{\# 2}\right) a_{\beta}, \quad 1 \leq|\gamma| \leq M_{0},
$$

vanishes where $y^{\# 2}$ is another $y$ value independent of $y^{\# 1}$. Only if all $g_{\gamma}$ vanish simultaneously the solution of (77) is independent of $y$. In this case $\lambda=r^{2}+1$ is an eigenvalue and the $a_{\beta}$ 's are the coefficients of the Fourier expansion of the corresponding Maass cusp form.

The probability to find an $r$ value such that all $g_{\gamma}$ vanish simultaneously is zero, because the discrete eigenvalues are of measure zero in the real numbers. Therefore, we make use of the intermediate value theorem where we look for simultaneous sign changes in $g_{\gamma}$. Once we have found them in at least half of the $g_{\gamma}$ 's we have found an interval which contains an eigenvalue with high probability. By some bisection and interpolation we can see if this interval really contains an eigenvalue, and by nesting up the interval until its size tends to zero we obtain the eigenvalue.

In order not to miss eigenvalues which lie close together nor to waste CPU time with a too fine grid, we use the adaptive $r$ grid introduced in 38.

\section{Eigenvalues for the Picard group}

We have found 13950 eigenvalues of the Laplacian for the Picard group in the interval $1<\lambda=r^{2}+1 \leq 19601$. 4115 of them belong to eigenfunctions of the symmetry class $\mathbf{D}, 2805$ to $\mathbf{G}, 3715$ to $\mathbf{C}$, and 3315 to $\mathbf{H}$. The smallest eigenvalue is $\lambda=r^{2}+1$ with $r=6.6221193402528$ which is in agreement with the lower bound $\lambda>\frac{2 \pi^{2}}{3}$ [37. Table $\square$ shows the first few eigenvalues of 
Table 1. The first few eigenvalues of the Laplacian for the Picard group. Listed is $r$. related to the eigenvalues via $\lambda=r^{2}+1$.

D

8.55525104

11.10856737

12.86991062

14.07966049

15.34827764

15.89184204

17.33640443

17.45131992

17.77664065

19.06739052

19.22290266

19.41119126

20.00754583

20.70798880

20.81526852

21.42887079

22.12230276

22.63055256

22.96230105

23.49617692

23.52784503

23.88978413

24.34601664

24.57501426

24.70045917

25.47067539

25.50724616

25.72392169
G

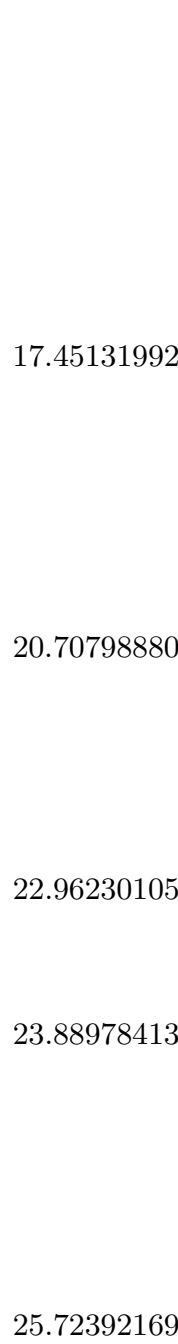

C

6.62211934

10.18079978

12.11527484

12.87936900

14.14833073

14.95244267

16.20759420

16.99496892

17.86305643

18.24391070

18.83298996

19.43054310

20.30030720

20.60686743

21.37966055

21.44245892

21.83248972

22.58475297

22.85429195

23.49768305

23.84275866

23.89515755

24.42133829

25.03278076

25.42905483

25.77588591

26.03903968

26.12361823
H

12.11527484

14.95244267

16.99496892

17.86305643

19.43054310

20.30030720

21.37966055

21.83248972

22.58475297

23.49768305

23.89515755

24.42133829

25.03278076

25.77588591

26.12361823 
each symmetry class. They agree with those of Steil [32] up to five decimal places. We next regard the statistics of the eigenvalues. First, we compare the output of our algorithm with Weyl's law and higher order corrections drawn from 39. This serves as a check whether we have found all eigenvalues. We then find it necessary to correct one of the terms in 39 numerically. Finally, we regard the spectral fluctuations and find that the nearest-neighbor spacing distribution closely resembles that of a Poisson random process as predicted by [9, 10, 11] and previously observed by 32].

In the first step we consider the level counting function

$$
N(r)=\#\left\{i \mid r_{i} \leq r\right\}
$$

and split it into two parts

$$
N(r)=\bar{N}(r)+N_{\text {fluc }}(r) .
$$

Here $\bar{N}$ is a smooth function describing the average increase in the number of levels and $N_{\text {fluc }}$ describes the fluctuations around the mean such that

$$
\lim _{R \rightarrow \infty} \frac{1}{R} \int_{1}^{R} N_{f l u c}(r) d r=0 .
$$

The average increase in the number of levels is given by Weyl's law 40, 41] and higher order corrections have been calculated by Matthies [39]. She obtained

$$
\bar{N}(r)=\frac{\operatorname{vol}(\mathcal{F})}{6 \pi^{2}} r^{3}+a_{2} r \log r+a_{3} r+a_{4}
$$

with the constants

$$
\begin{aligned}
& a_{2}=-\frac{3}{2 \pi}, \\
& a_{3}=\frac{1}{\pi}\left[\frac{13}{16} \log 2+\frac{7}{4} \log \pi-\log \Gamma\left(\frac{1}{4}\right)+\frac{2}{9} \log (2+\sqrt{3})+\frac{3}{2}\right], \\
& a_{4}=-\frac{1}{2} .
\end{aligned}
$$

We compare our results for $N(r)$ with (8) by defining

$$
N_{\text {fluc }}(r)=N(r)-\bar{N}(r) .
$$

$N_{\text {fluc }}$ fluctuates around zero or a negative integer whose absolute value gives the number of missing eigenvalues, see figure 9 Unfortunately, our algorithm does not find all eigenvalues in one single run. In the first run it finds about $97 \%$ of the eigenvalues. Apart from very few exceptions the remaining eigenvalues are found in the third run. To be more specific, we plotted $N_{\text {fluc }}$ decreased by $\frac{1}{2}$, because $N(r)-\bar{N}(r)$ is approximately $\frac{1}{2}$ whenever $\lambda=r^{2}+1$ is an eigenvalue. Furthermore, we took the eigenvalue $\lambda=0$ into account. We remark that we never find more eigenvalues than predicted by (8). A plot indicating that $N_{\text {fluc }}$ fluctuates around zero is shown in figure 10 where we plotted the integral 


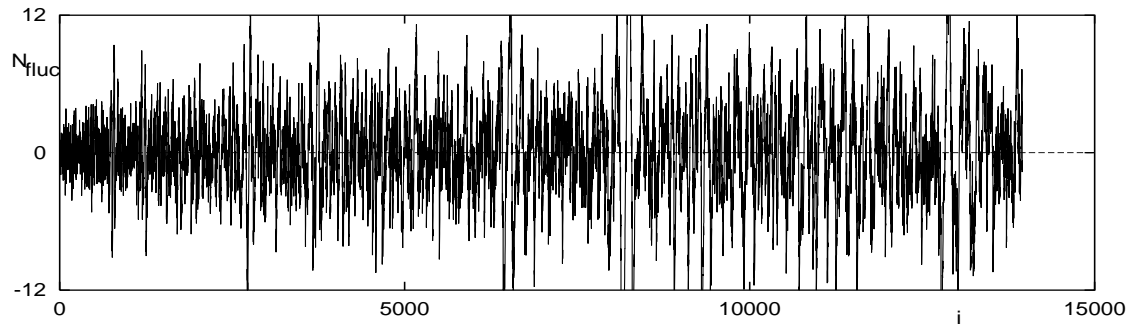

Fig. 9. $N_{\text {fluc }}\left(r_{i}\right)$ as a function of $i$ fluctuating around zero.

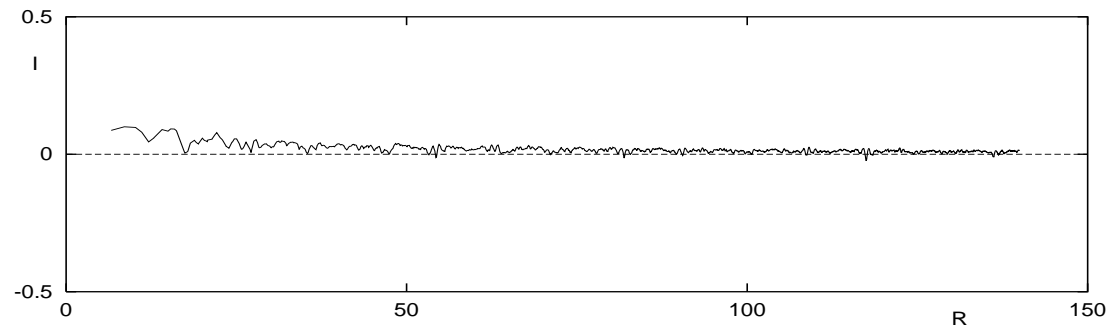

Fig. 10. $I$ as a function of $R$ showing that $I \stackrel{R \rightarrow \infty}{\longrightarrow} 0$.

$$
I(R)=\frac{1}{R} \int_{1}^{R} N_{f l u c}(r) d r .
$$

So far, everything seems to be consistent. Taking the desymmetrized spectra into account (8) is modified 39

$$
\bar{N}(r)=\frac{\operatorname{vol}(\mathcal{F})}{24 \pi^{2}} r^{3}+b_{1} r^{2}+b_{2} r \log r+b_{3} r+b_{4}
$$

with the constants depending on the symmetry class. For the symmetry class $\mathbf{D}$ the constants are given in 39 as

$$
\begin{aligned}
& b_{1}= \frac{1}{24}, \\
& b_{2}=-\frac{13}{8 \pi}, \\
& b_{3}= \frac{1}{4 \pi}\left[-\frac{11}{16} \log 2+\frac{19}{4} \log \pi-\log \Gamma\left(\frac{1}{4}\right)\right. \\
&\left.\quad+\frac{2}{9} \log (2+\sqrt{3})+\frac{1}{4} \log (3+2 \sqrt{2})+\frac{13}{2}\right], \\
& b_{4}=-\frac{47}{72} .
\end{aligned}
$$

\section{For $\mathbf{G}$}




$$
\begin{aligned}
b_{1}= & -\frac{1}{24}, \\
b_{2}= & \frac{3}{8 \pi}, \\
b_{3}= & \frac{1}{4 \pi}\left[\frac{37}{16} \log 2+\frac{3}{4} \log \pi-\log \Gamma\left(\frac{1}{4}\right)\right. \\
& \left.\quad+\frac{2}{9} \log (2+\sqrt{3})+\frac{1}{4} \log (3+2 \sqrt{2})-\frac{3}{2}\right], \\
b_{4}= & -\frac{25}{72} .
\end{aligned}
$$

For $\mathbf{C}$

$$
\begin{aligned}
b_{1}= & \frac{1}{96}, \\
b_{2}= & -\frac{1}{8 \pi}, \\
b_{3}= & \frac{1}{4 \pi}\left[\frac{5}{16} \log 2+\frac{3}{4} \log \pi-\log \Gamma\left(\frac{1}{4}\right)\right. \\
& \left.\quad+\frac{2}{9} \log (2+\sqrt{3})-\frac{1}{4} \log (3+2 \sqrt{2})+\frac{1}{2}\right], \\
b_{4}= & \frac{125}{576} .
\end{aligned}
$$

And for $\mathbf{H}$

$$
\begin{aligned}
b_{1}= & -\frac{1}{96}, \\
b_{2}= & -\frac{1}{8 \pi}, \\
b_{3}= & \frac{1}{4 \pi}\left[\frac{21}{16} \log 2+\frac{3}{4} \log \pi-\log \Gamma\left(\frac{1}{4}\right)\right. \\
& \left.\quad+\frac{2}{9} \log (2+\sqrt{3})-\frac{1}{4} \log (3+2 \sqrt{2})+\frac{1}{2}\right], \\
b_{4}= & \frac{163}{576} .
\end{aligned}
$$

Let $\left\{r_{i}\right\}$ be a sequence related to the consecutive eigenvalues $\lambda=r^{2}+1$. If we plot $N_{\text {fluc }}\left(r_{i}\right)$ as a function of $i$ for the desymmetrized spectra we obtain small deviations which can hardly be seen in figure 11] But if we plot the integral (10) we see that $N_{\text {fluc }}$ does not really fluctuate around zero. Instead, in figure 12 we see systematic deviations, but the discrepancy is much less than one eigenvalue for each symmetry class. Since the number of eigenvalues is integervalued we do not assume that we have found less or too many eigenvalues. Therefore, we fit the constants $b_{1}, b_{2}, b_{3}, b_{4}$ in (11) and obtain new constants for each of the symmetry classes. Since the integrals $I(R)$ in figure 12 show a linear behavior, the constants $b_{1}$ and $b_{2}$ seem to be correct. We thus only change the constants $b_{3}$ and $b_{4}$ by fitting them numerically. For the symmetry class $\mathbf{D}$ the new constants are

$$
\begin{array}{ll}
b_{3}=0.8639 \ldots & \text { instead of } b_{3}=0.8679 \ldots, \\
b_{4}=-0.288 \ldots & \text { instead of } b_{4}=-0.653 \ldots .
\end{array}
$$

For $\mathbf{G}$

$$
\begin{array}{ll}
b_{3}=0.0285 \ldots & \text { instead of } b_{3}=0.0324 \ldots, \\
b_{4}=-0.184 \ldots & \text { instead of } b_{4}=-0.347 \ldots .
\end{array}
$$




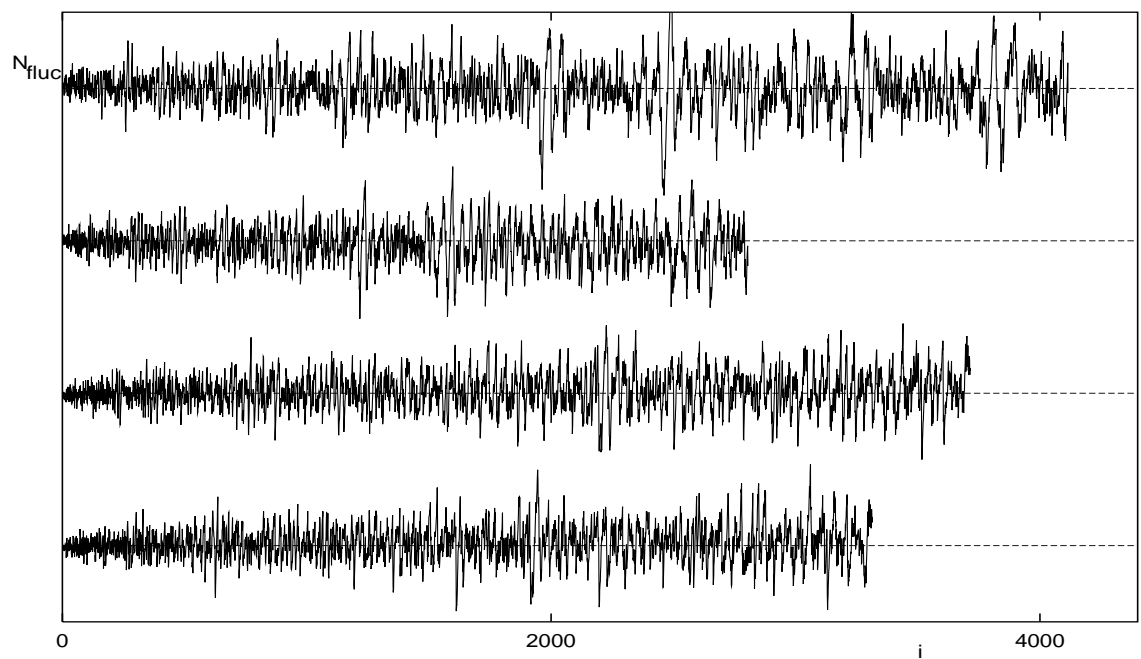

Fig. 11. $N_{\text {fluc }}\left(r_{i}\right)$ as a function of $i$ for each symmetry class. The symmetry classes are $\mathbf{D}, \mathbf{G}, \mathbf{C}, \mathbf{H}$ from top to bottom.

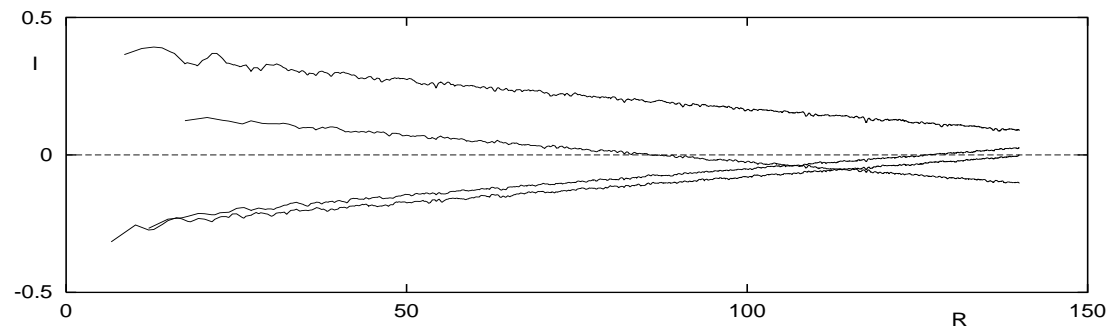

Fig. 12. $I$ as a function of $R$ showing the systematic deviations from $I \stackrel{R \rightarrow \infty}{\longrightarrow} 0$. Each curve belongs to one of the symmetry classes $\mathbf{D}, \mathbf{G}, \mathbf{C}, \mathbf{H}$.

\section{For C}

$$
\begin{array}{ll}
b_{3}=0.0150 \ldots \\
b_{4}=-0.062 \ldots
\end{array} \quad \begin{aligned}
& \text { instead of } b_{3}=0.0111 \ldots, \\
& \text { instead of } b_{4}=0.217 \ldots
\end{aligned}
$$

And $\mathbf{H}$

$$
\begin{array}{ll}
b_{3}=0.0702 \ldots & \text { instead of } b_{3}=0.0662 \ldots, \\
b_{4}=0.034 \ldots & \text { instead of } b_{4}=0.283 \ldots .
\end{array}
$$

In figure 13 we present the integral (10) with the corrected constants.

Now we are able to regard the spectral fluctuations. We unfold the spectrum,

$$
x_{i}=\bar{N}\left(r_{i}\right)
$$




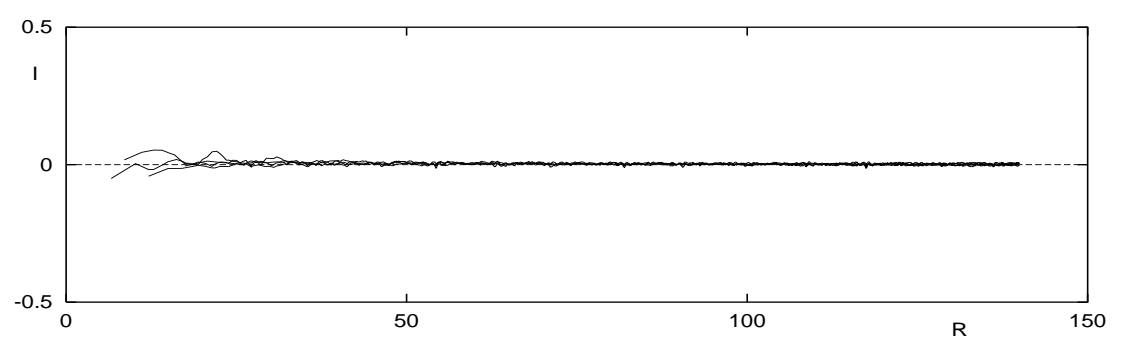

Fig. 13. $I$ as a function of $R$ with the corrected constants. Each curve belongs to one of the symmetry classes. The curves are quite indistinguishable from 0 .

in order to obtain rescaled eigenvalues $x_{i}$ with a unit mean density. Then

$$
s_{i}=x_{i+1}-x_{i}
$$

defines the sequence of nearest-neighbor level spacings which has a mean value of 1 as $i \rightarrow \infty$. We find that the spacing distribution comes close to that of a Poisson random process,

$$
P_{\text {Poisson }}(s)=\mathrm{e}^{-s}
$$

see figures 14 to 17 as opposed to that of a Gaussian orthogonal ensemble of random matrix theory,

$$
P_{\mathrm{GOE}}(s) \simeq \frac{\pi}{2} s \mathrm{e}^{-\frac{\pi}{4} s^{2}} .
$$

The integrated distribution,

$$
\mathcal{I}(s)=\int_{0}^{s} P(t) d t
$$

showing the fraction of spacings up to a given length is also shown in figures 14 to 17 The spacing distributions of the desymmetrized spectra are in accordance with the conjecture of arithmetic quantum chaos. Also in agreement with the conjecture is that we have not found any degenerate eigenvalues within each symmetry class. But taking the eigenvalues of all four symmetry classes together systematic degeneracies occur due to the following:

Theorem 1 (Steil [32]). If $\lambda=r^{2}+1$ is an eigenvalue corresponding to an eigenfunction of the symmetry class $\mathbf{G}$ resp. $\mathbf{H}$ then there exists an eigenfunction of the symmetry class $\mathbf{D}$ resp. $\mathbf{C}$ corresponding to the same eigenvalue.

These degeneracies were first observed by Huntebrinker [30] and later explained by Steil [32] with the use of the Hecke operators [29, 33]. The Hecke operators are defined by 


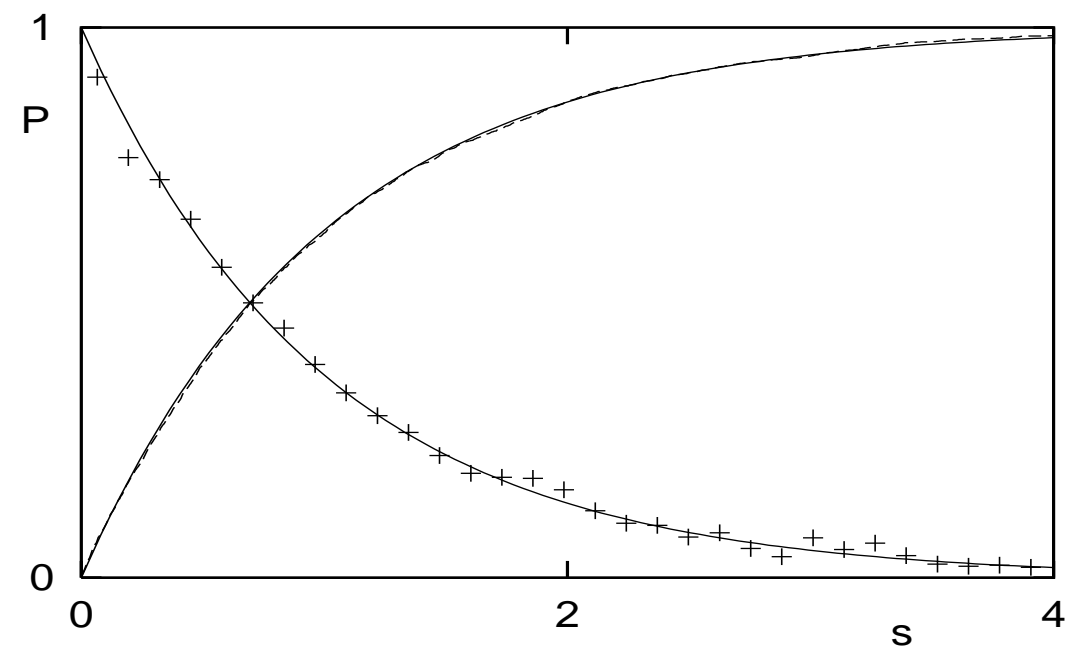

Fig. 14. Level spacing distribution for the symmetry class D. The abscissa displays the spacings $s$. The dashed curve starting at the origin is the integrated distribution. For comparison, the full curves show a Poisson distribution.

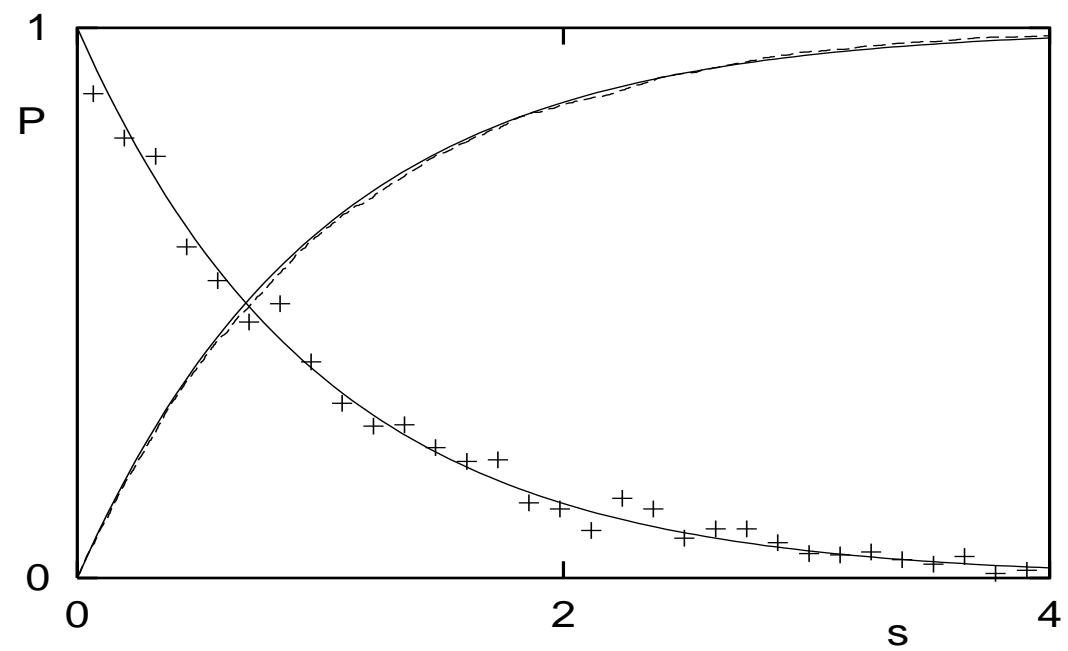

Fig. 15. Level spacing distribution for the symmetry class $\mathbf{G}$. 


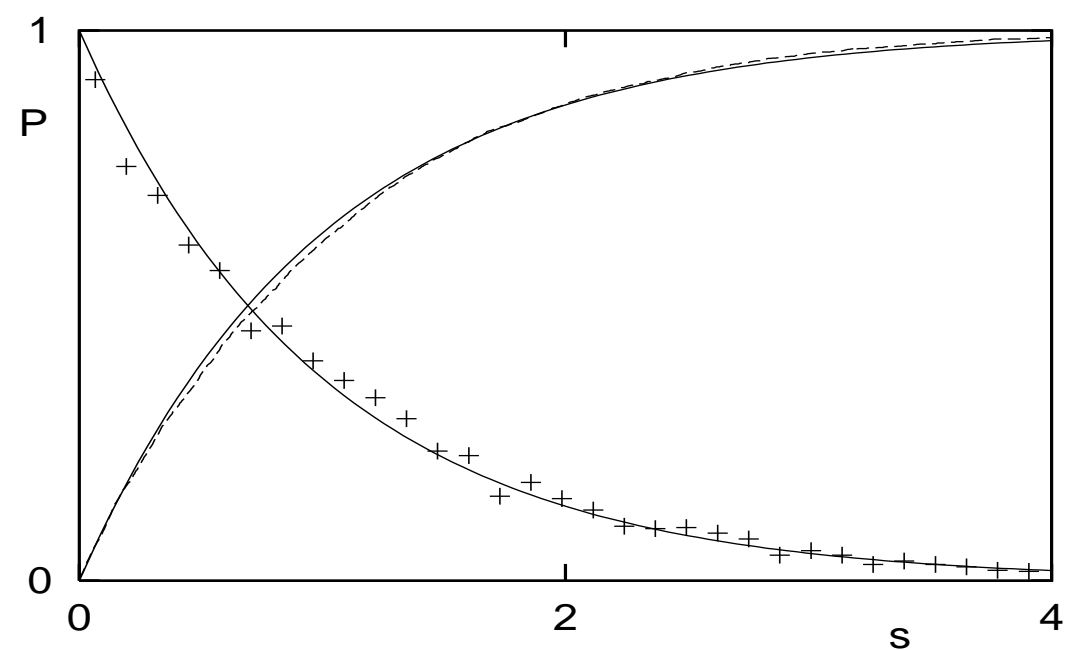

Fig. 16. Level spacing distribution for the symmetry class $\mathbf{C}$.

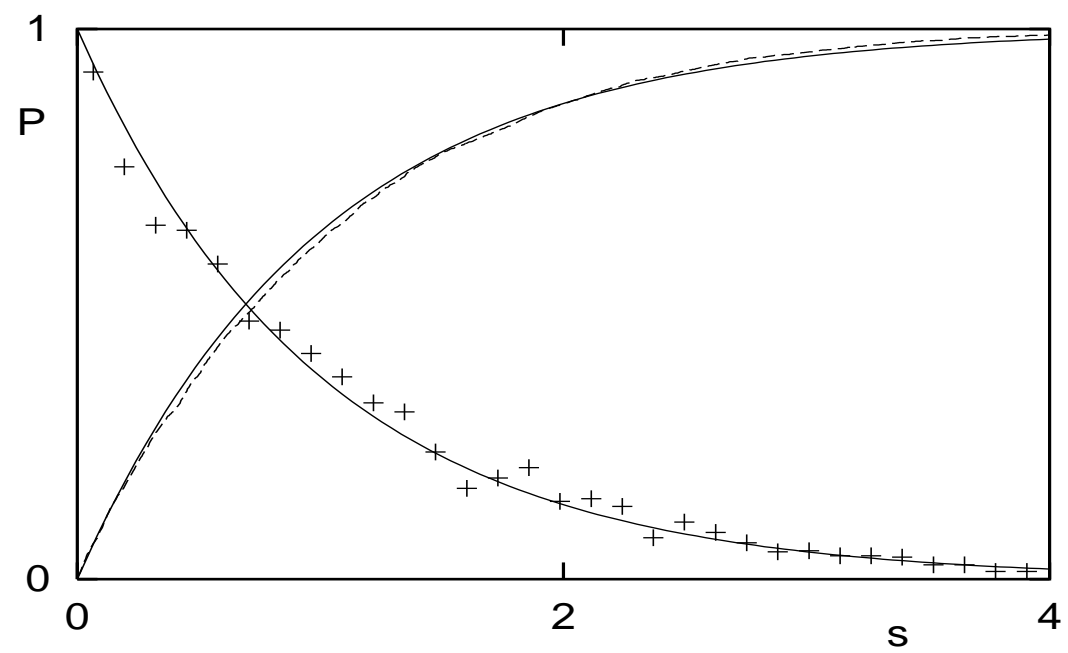

Fig. 17. Level spacing distribution for the symmetry class $\mathbf{H}$. 


$$
\mathrm{T}_{\gamma} g(z)=\frac{1}{|\gamma|} \sum_{\substack{a, b, d \in \mathbb{Z}[\mathrm{i}]-\{0\} \\ a d=\gamma \\ b(\bmod d) \\ \Re d>0, \Im d \geq 0 .}} g\left((a d)^{-\frac{1}{2}}(a z+b)(d)^{-1}(a d)^{\frac{1}{2}}\right), \quad \gamma \in \mathbb{Z}[\mathrm{i}]-\{0\} .
$$

They are self adjoint operators which commute with the Laplacian and among each other. One can therefore simultaneously diagonalize these operators. The corresponding Maass cusp forms are then called Hecke eigenfunctions. The eigenvalue equation of the Hecke operators reads

$$
\mathrm{T}_{\gamma} g_{r}(z)=t_{\gamma} g_{r}(z), \quad \gamma \in \mathbb{Z}[\mathrm{i}]-\{0\},
$$

where each Hecke eigenfunction is either identical to a Maass cusp form with a given symmetry or to a superposition of Maass cusp forms corresponding to the same eigenvalue $\lambda=r^{2}+1$, but to different symmetry classes,

$$
g_{r}(z)=\sum_{\substack{n \in \mathbb{N} \\\left(\Delta+\left(r^{2}+1\right)\right) f_{n}(z)=0}} c_{n} f_{n}(z)
$$

The Hecke operators are multiplicative,

$$
\mathrm{T}_{\gamma} \mathrm{T}_{\beta} g_{r}(z)=\sum_{d \mid(\gamma, \beta)} \mathrm{T}_{\frac{\gamma \beta}{d^{2}}} g_{r}(z),
$$

and the Hecke eigenvalues are connected to the Fourier coefficients,

$$
b_{\gamma}=b_{1} t_{\gamma}, \quad \gamma \in \mathbb{Z}[\mathrm{i}]-\{0\},
$$

where the Fourier coefficients $b_{\gamma}$ of the Hecke eigenfunctions are given by

$$
b_{\gamma}=\sum_{n} c_{n} a_{\gamma, n}
$$

and the index $n$ at the Fourier coefficients of the Maass cusp forms $a_{\gamma}=a_{\gamma, n}$ means that they belong to the Fourier expansion of the $n$-th Maass cusp form $f_{n}(z)$.

Lemma 1 (Steil [32]). If $g_{r}(z)$ is a Hecke eigenfunction that does not vanish identically, then:

(i) Its first Fourier coefficient is never zero, $b_{1} \neq 0$.

(ii) A Hecke eigenfunction cannot be of symmetry class $\mathbf{G}$ or $\mathbf{H}$.

(iii) Hecke eigenfunctions can always be desymmetrized such that they fall either into the symmetry class $\mathbf{D} \cup \mathbf{G}$ or $\mathbf{C} \cup \mathbf{H}$.

Proof (Steil's theorem). Let $f_{n}(z)$ be a Maass cusp form of the symmetry class G or H. Due to Steil's lemma, it cannot be a Hecke eigenfunction. Since one can diagonalize the Laplacian and the Hecke operators simultaneously, there 
have to exist linearly independent Maass cusp forms $f_{n+k}, k=0, \ldots, K$ corresponding to the same eigenvalue $\lambda=r^{2}+1$ such that

$$
\sum_{k=0}^{K} c_{n+k} f_{n+k}(z)=g_{r}(z)
$$

is a Hecke eigenfunction.

At least one of these Maass cusp forms has to be of the symmetry class $\mathbf{D}$ or $\mathbf{C}$ in order that

$$
b_{1}=\sum_{k=0}^{K} c_{n+k} a_{1, n+k}
$$

does not vanish.

Since the Hecke eigenfunctions can be desymmetrized such that they fall into the symmetry class $\mathbf{D} \cup \mathbf{G}$ resp. $\mathbf{C} \cup \mathbf{H}$ they are a superposition of either Maass cusp forms of the symmetry classes $\mathbf{D}$ and $\mathbf{G}$ or of Maass cusp forms of the symmetry classes $\mathbf{C}$ and $\mathbf{H}$. Therefore, if $f_{n}(z)$ is of symmetry class $\mathbf{G}$ one of the $f_{n+k}, k=1, \ldots, K$ is of symmetry class $\mathbf{D}$, and if $f_{n}(z)$ is of symmetry class $\mathbf{H}$ one of the $f_{n+k}, k=1, \ldots, K$ is of symmetry class $\mathbf{C}$.

Based on our numerical results we now conjecture the following:

Conjecture 4. Taking all four symmetry classes together, there are no degenerate eigenvalues other than those explained by Steil's theorem. Furthermore, the degenerate eigenvalues which are explained by Steil's theorem occur only in pairs of two degenerate eigenvalues. They never occur in pairs of three or more degenerate eigenvalues.

Maass cusp forms of the symmetry classes $\mathbf{G}$ and $\mathbf{H}$ indeed occur. On the one hand we have found a number of them numerically. On the other hand, Weyl's law also explains their existence. Due to this the number of eigenvalues whose corresponding Maass cusp forms belong to a specific symmetry class is in leading order independent of the choice of the symmetry class. Weyl's law together with Steil's theorem lead to the following:

Conjecture 5. The sequence of non-degenerate eigenvalues in the spectrum of the Laplacian for the Picard group is of density zero.

This means that as $\lambda \rightarrow \infty$

$$
\frac{\#\{\text { non-degenerate eigenvalues } \leq \lambda\}}{\#\{\text { degenerate eigenvalues } \leq \lambda\}} \rightarrow 0 .
$$

Table 1 looks as if it would contradict this conjecture. But this is due to the fact that only the first few eigenvalues are listed. In table 2 we list some consecutive large eigenvalues where we can see a better agreement with the conjecture. 
Table 2. Some consecutive large eigenvalues of the Laplacian for the Picard group. Listed is $r$, related to the eigenvalues via $\lambda=r^{2}+1$.

$\begin{array}{cccc}\mathbf{D} & \mathbf{G} & \mathbf{C} & \mathbf{H} \\ & & & \\ 139.65419675 & 139.65419675 & 139.66399548 & 139.66399548 \\ 139.65434417 & 139.65434417 & 139.66785333 & 139.66785333 \\ 139.65783548 & 139.65783548 & 139.66922266 & 139.66922266 \\ 139.66104047 & 139.66104047 & 139.67870460 & 139.67870460 \\ 139.67694018 & & 139.68234200 & 139.68234200 \\ 139.68162707 & 139.68162707 & 139.68424704 & 139.68424704 \\ 139.68657976 & & 139.69369972 & 139.69369972 \\ 139.71803029 & 139.71803029 & 139.69413379 & 139.69413379 \\ 139.72166907 & 139.72166906 & 139.69657741 & 139.69657741 \\ 139.78322452 & 139.78322452 & 139.73723373 & 139.73723373 \\ 139.81928622 & 139.81928622 & 139.73828541 & 139.73828541 \\ 139.81985670 & 139.81985670 & 139.74467774 & 139.74467774 \\ 139.82826034 & 139.82826034 & 139.75178180 & 139.75178180 \\ 139.84250751 & & 139.75260292 & 139.75260292 \\ 139.87781072 & 139.87781072 & 139.79620628 & 139.79620628 \\ 139.87805540 & & 139.80138072 & 139.80138072 \\ 139.88211647 & 139.88211647 & 139.81243991 & 139.81243991 \\ 139.91782003 & 139.91782003 & 139.81312982 & 139.81312982 \\ 139.91893517 & & 139.82871870 & 139.82871870 \\ 139.92397167 & 139.92397167 & 139.86401372 & 139.86401372 \\ 139.92721861 & 139.92721861 & 139.86461581 & 139.86461581 \\ 139.93117207 & 139.93117207 & 139.89407865 & 139.89407865 \\ 139.93149277 & 139.93149277 & 139.89914777 & 139.89914777 \\ 139.94067283 & & 139.90090849 & 139.90090849 \\ 139.94396890 & 139.94396890 & 139.91635302 & 139.91635302 \\ 139.95074070 & & 139.94071729 & 139.94071729 \\ 139.95124805 & 139.95124805 & 139.95080198 & 139.95080198 \\ 139.99098324 & 139.99098324 & 139.97043676 & 139.97043676\end{array}$




\section{Summary}

Our principal goal was to test the conjecture of arithmetic quantum chaos numerically in one example. For this purpose we have chosen a point particle moving freely in the three-dimensional and negatively curved quotient space of the Picard group. Identifying the solutions of the stationary Schrödinger equation with Maass waveforms allowed us to use Hejhal's algorithm to compute the eigenfunctions and eigenvalues numerically. Having computed 13950 eigenvalues (and eigenfunctions), which exceeds all previous computations in non-integrable three-dimensional systems, we demonstrated that our numerical results are in accordance with the conjecture of arithmetic quantum chaos. Within each symmetry class we do not find any degenerate eigenvalues, but taking all four symmetry classes together, almost all eigenvalues become degenerate in the limit of large eigenvalues $\lambda \rightarrow \infty$. This behaviour was explained by the interplay of the symmetries with the Hecke-operators.

\section{Acknowledgments}

The help of Ralf Aurich, Jens Bolte, Dennis A. Hejhal and Frank Steiner is gratefully acknowledged. The author is supported by the Deutsche Forschungsgemeinschaft under the contract no. DFG Ste 241/16-1. Part of the work was done while I was a member of Dennis A. Hejhal's group in Uppsala (Sweden) supported by the European Commission Research Training Network HPRN-CT-2000-00103. The computations were run on the UniversitätsRechenzentrum Ulm.

\section{A The K-Bessel function}

The K-Bessel function is defined by

$$
K_{\mathrm{i} r}(x)=\int_{0}^{\infty} \mathrm{e}^{-x \cosh t} \cos (r t) d t, \quad \Re x>0, \quad r \in \mathbb{C},
$$

see Watson [42, and is real for real arguments $x$ and real or imaginary order ir. It solves the modified Bessel differential equation

$$
x^{2} u^{\prime \prime}(x)+x u^{\prime}(x)-\left(x^{2}-r^{2}\right) u(x)=0,
$$

and decays exponentially for large arguments

$$
K_{\mathrm{i} r}(x) \sim \sqrt{\frac{\pi}{2 x}} \mathrm{e}^{-x} \text { for } x \rightarrow \infty .
$$

A second linearly independent solution of the modified Bessel differential equation is the I-Bessel function 


$$
I_{\mathrm{i} r}(x)=\left(\frac{x}{2}\right)^{\mathrm{i} r} \sum_{k=0}^{\infty} \frac{\left(\frac{x}{2}\right)^{2 k}}{k ! \Gamma(\mathrm{i} r+k+1)},
$$

which grows exponentially for large arguments

$$
I_{\mathrm{i} r}(x) \sim \sqrt{\frac{1}{2 \pi x}} \mathrm{e}^{x} \quad \text { for } x \rightarrow \infty .
$$

The K-Bessel function decreases exponentially when $r$ increases. This can be compensated by multiplication with the factor $\mathrm{e}^{\frac{\pi r}{2}}$.

In order to compute the K-Bessel function numerically for small or moderate imaginary order we use its continued fraction representation which follows from the Miller algorithm 43 .

\section{References}

1. M. V. Berry and M. Tabor. Closed orbits and the regular bound spectrum. Proc. Roy. Soc. London Ser. A, 349:101-123, 1976.

2. O. Bohigas, M.-J. Giannoni, and C. Schmit. Characterization of chaotic quantum spectra and universality of level fluctuation laws. Phys. Rev. Lett., 52:1-4, 1984.

3. O. Bohigas, M.-J. Giannoni, and C. Schmit. Spectral fluctuations, random matrix theories and chaotic motion. Stochastic processes in classical and quantum systems. Lecture Notes in Phys., 262:118-138, 1986.

4. M. L. Mehta. Random matrices. Academic Press, second edition, 1991.

5. R. Aurich and F. Steiner. On the periodic orbits of a strongly chaotic system. Physica D, 32:451-460, 1988.

6. R. Aurich, E. B. Bogomolny, and F. Steiner. Periodic orbits on the regular hyperbolic octagon. Physica D, 48:91-101, 1991.

7. R. Aurich and F. Steiner. Periodic-orbit sum rules for the Hadamard-Gutzwiller model. Physica D, 39:169-193, 1989.

8. R. Aurich and F. Steiner. Energy-level statistics of the Hadamard-Gutzwiller ensemble. Physica D, 43:155-180, 1990.

9. E. B. Bogomolny, B. Georgeot, M.-J. Giannoni, and C. Schmit. Chaotic billiards generated by arithmetic groups. Phys. Rev. Lett., 69:1477-1480, 1992.

10. J. Bolte, G. Steil, and F. Steiner. Arithmetical chaos and violation of universality in energy level statistics. Phys. Rev. Lett., 69:2188-2191, 1992.

11. P. Sarnak. Arithmetic quantum chaos. Israel Math. Conf. Proc., 8:183-236, 1995.

12. A. Borel. Introduction aux groupes arithmétiques. (French). Hermann, 1969.

13. A. Terras. Harmonic Analysis on Symmetric Spaces and Applications, volume 1. Springer, 1985.

14. H. Maaß. Über eine neue Art von nichtanalytischen automorphen Funktionen und die Bestimmung Dirichletscher Reihen durch Funktionalgleichungen. (German). Math. Ann., 121:141-183, 1949.

15. A. Selberg. Harmonic analysis and discontinuous groups in weakly symmetric Riemannian spaces with applications to Dirichlet series. J. Indian Math. Soc., 20:47-87, 1956. 
16. W. Roelcke. Das Eigenwertproblem der automorphen Formen in der hyperbolischen Ebene, I. (German). Math. Ann., 167:292-337, 1966.

17. W. Roelcke. Das Eigenwertproblem der automorphen Formen in der hyperbolischen Ebene. II. (German). Math. Ann., 168:261-324, 1967.

18. L. D. Faddeev. Expansion in eigenfunctions of the Laplace operator on the fundamental domain of a discrete group on the Lobačevskiǐ plane. Transactions of the Moscow Math. Soc., 17:357-386, 1967.

19. G. Shimura. Introduction to the Arithmetic Theory of Automorphic Functions. Princeton Univ. Press, 1971.

20. D. A. Hejhal. The Selberg trace formula for $\operatorname{PSL}(2, \mathbb{R})$. Lecture Notes in Math. 1001. Springer, 1983.

21. T. Miyake. Modular forms. Springer, 1989.

22. A. B. Venkov. Spectral Theory of Automorphic Functions and Its Applications. Kluwer Academic Publishers, 1990.

23. H. Iwaniec. Introduction to the Spectral Theory of Automorphic Forms. Revista Matemática Iberoamericana, 1995.

24. G. Humbert. Sur la mesure des classes d'Hermite de discriminant donné dans un corps quadratique imaginaire, et sur certaines volumes non euclidiens. (French). C. R. Acad. Sci. Paris, 169:448-454, 1919.

25. H. Maaß. Automorphe Funktionen von mehreren Veränderlichen und Dirichletsche Reihen. (German). Abh. Math. Semin. Univ. Hamb., 16:72-100, 1949.

26. T. Kubota. Elementary Theory of Eisenstein Series. Kodansha, Tokyo and Halsted Press, 1973.

27. J. Elstrodt, F. Grunewald, and J. Mennicke. Eisenstein series on threedimensional hyperbolic space and imaginary quadratic number fields. J. Reine Angew. Math., 360:160-213, 1985.

28. J. Elstrodt, F. Grunewald, and J. Mennicke. Groups Acting on Hyperbolic Space. Springer, 1998.

29. M. N. Smotrov and V. V. Golovčanskiǐ. Small eigenvalues of the Laplacian on $\Gamma \backslash H_{3}$ for $\Gamma=P S L_{2}(\mathbb{Z}[i])$. Preprint, 91-040, Bielefeld 1991.

30. W. Huntebrinker. Numerical computation of eigenvalues of the Laplace-Beltrami operator on three-dimensional hyperbolic spaces by finite-element methods. Diss. Summ. Math., 1:29-36, 1996.

31. F. Grunewald and W. Huntebrinker. A numerical study of eigenvalues of the hyperbolic Laplacian for polyhedra with one cusp. Experiment. Math., 5:57-80, 1996.

32. G. Steil. Eigenvalues of the Laplacian for Bianchi groups. In D. A. Hejhal, J. Friedman, M. C. Gutzwiller, and A. M. Odlyzko, editors, Emerging applications of number theory, IMA Series No. 109, pages 617-641. Springer, 1999.

33. D. Heitkamp. Hecke-Theorie zur SL $(2 ; \mathfrak{o})$. (German). Schriftenreihe des Mathematischen Instituts der Universität Münster, 3. Serie, 5, 1992.

34. D. A. Hejhal. On eigenfunctions of the Laplacian for Hecke triangle groups. In D. A. Hejhal, J. Friedman, M. C. Gutzwiller, and A. M. Odlyzko, editors, Emerging applications of number theory, IMA Series No. 109, pages 291-315. Springer, 1999.

35. B. Selander and A. Strömbergsson. Sextic coverings of genus two which are branched at three points. UUDM report 2002:16, Uppsala 2002.

36. H. Avelin. Research announcement on the deformation of cusp forms. UUDM report 2002:26, Uppsala 2002. 
37. K. Stramm. Kleine Eigenwerte des Laplace-Operators zu Kongruenzgruppen. (German). Schriftenreihe des Mathematischen Instituts der Universität Münster, 3. Serie, 11, 1994.

38. H. Then. Maaß cusp forms for large eigenvalues. Math. Comp., 74:363-381, 2005.

39. C. Matthies. Picards Billard. Ein Modell für Arithmetisches Quantenchaos in drei Dimensionen. (German). PhD thesis, Universität Hamburg, 1995.

40. H. Weyl. Das asymptotische Verteilungsgesetz der Eigenwerte linearer partieller Differentialgleichungen. (German). Math. Ann., 71:441-479, 1912.

41. V. G. Avakumović. Über die Eigenfunktionen auf geschlossenen Riemannschen Mannigfaltigkeiten. (German). Math. Z., 65:327-344, 1956.

42. G. N. Watson. A treatise on the theory of Bessel functions. Cambridge University Press, 1944.

43. N. M. Temme. On the numerical evaluation of the modified Bessel function of the third kind. J. Comput. Phys., 19:324-337, 1975. 